\title{
TAIL ESTIMATES FOR HOMOGENIZATION THEOREMS IN RANDOM MEDIA
}

\author{
DANIEL BOIVIN ${ }^{1}$
}

\begin{abstract}
Consider a random environment in $\mathbb{Z}^{d}$ given by i.i.d. conductances. In this work, we obtain tail estimates for the fluctuations about the mean for the following characteristics of the environment: the effective conductance between opposite faces of a cube, the diffusion matrices of periodized environments and the spectral gap of the random walk in a finite cube.
\end{abstract}

Mathematics Subject Classification. 60K37, 35B27, 82B44.

Received April 13, 2007. Revised October 17, 2007.

\section{INTRODUCTION}

Consider the $d$-dimensional cubic lattice $\mathbb{Z}^{d}, d \geq 2$, as a random electrical network. The conductances of the edges $e$ of $\mathbb{Z}^{d}$ are given by a sequence of i.i.d. positive random variables $\left(a(e, \omega) ; e\right.$ is an edge of $\left.\mathbb{Z}^{d}\right)$ on a probability space $(\Omega, \mathcal{F}, \mathbb{P})$ interpreted as the space of the environments.

The effective conductance of the electrical network between two opposite faces of the cube $[0, N+1]^{d} \cap \mathbb{Z}^{d}$, $N>2$ is the strength of the electrical flow through one of the faces needed to maintain a unit potential difference between the two opposite faces. It will be denoted by $\mathcal{C}_{N}$. See [13] Section 13.2 for a survey of some results and open questions about the asymptotic properties of the effective conductance.

We say that the conductances are uniformly elliptic if there is a constant $\kappa \geq 1$, called the ellipticity constant, such that $\mathbb{P}$ - a.s., for all edges $e$ of $\mathbb{Z}^{d}$,

$$
\kappa^{-1} \leq a(e, \omega) \leq \kappa .
$$

Using the basic laws of electric networks (see for example [24] Chap. 8), one can calculate that if all the edges of $Q_{N}$ have unit conductance then the effective conductance between opposite faces is $N^{d-1} /(N+1)$. Therefore by the variational representation of the effective conductance (12), or by the monotonicity law [24] Chapter 8 , if the conductances are uniformly elliptic then

$$
\kappa^{-1} \leq(N+1) N^{1-d} \mathcal{C}_{N} \leq \kappa .
$$

\footnotetext{
Keywords and phrases. Periodic approximation, random environments, fluctuations, effective diffusion matrix, effective conductance, non-uniform ellipticity.

${ }^{1}$ Laboratoire de Mathématiques UMR 6205, Université de Bretagne Occidentale, 6, avenue Le Gorgeu, CS 93837, 29238 Brest Cedex 3, France; boivin@univ-brest.fr 
Furthermore, Jikov et al. [16] Chapters 8 and 9 showed by homogenization methods that for some differential operators with uniformly elliptic and stationary coefficients and for some percolation models,

$$
A_{N}:=(N+1)^{2} N^{-d} \mathcal{C}_{N} \text { converges a.s. as } N \rightarrow \infty .
$$

See also [22],[4] and [2] Section 10.

A first result here is an estimate of the fluctuations of $A_{N}$ around its mean when the conductances are i.i.d. and uniformly elliptic. The convenience of working with $A_{N}$ rather that $\mathcal{C}_{N}$ is that we readily see for which dimensions the estimates are interesting. In particular, for i.i.d. and uniformly elliptic conductances, we see that although $A_{N}$ converges for all dimensions, our estimates are interesting only for dimensions $d \geq 3$.

Denote by $\mathcal{L}^{d}$ the set of edges of the cubic lattice $\mathbb{Z}^{d}$.

Theorem 1. Let $\left(a(e) ; e \in \mathcal{L}^{d}\right), d \geq 3$, be a sequence of i.i.d. uniformly elliptic conductances with ellipticity constant $\kappa$. Set $C_{0}=64 \kappa^{3}$.

Then for all $t \geq 0$ and $N \geq 1$,

$$
\mathbb{P}\left(\left|A_{N}-\mathbb{E} A_{N}\right| \geq t N^{(2-d) / 2}\right) \leq 4 \exp \left(-t / \sqrt{\kappa C_{0}}\right)
$$

and

$$
\operatorname{Var}\left(A_{N}\right) \leq \kappa C_{0} N^{2-d}
$$

where the expectation and the variance with respect to $\mathbb{P}$ are denoted by $\mathbb{E}$ and Var respectively.

In Theorem 3, tail estimates are obtained for environments given by the non uniformly elliptic conductances considered in Fontes and Mathieu [11]. They complement the lower bounds on the variance obtained by Wehr [28] for some distributions of the conductances.

Let us now use the random conductances to construct a reversible Markov chain $\left(X_{n} ; n \geq 0\right)$ on $\mathbb{Z}^{d}$. In the environment $\omega$, the probability transition between two neighbours $x, y \in \mathbb{Z}^{d}$, which will be denoted by $x \sim y$, is given by

$$
p(x, y, \omega)=a(x, y, \omega) / a(x, \omega), \quad x \sim y
$$

where $a(x, y, \omega)$ is the conductance of the edge joining $x$ and $y$ and $a(x, \omega)=\sum_{y \sim x} a(x, y, \omega)$. Note that the Markov chain is reversible with a stationary measure given by $a(x, \omega)$.

In [26] Section 1, Sidoravicius and Sznitman showed that if the conductances are stationary and uniformly elliptic then a functional central limit theorem holds in almost all environment. Weaker versions of the invariance principle already appeared in [19-21]. Let $\mathcal{D}_{0}$ be the diffusion matrix of the limiting Brownian motion. When the conductances are i.i.d., $\mathcal{D}_{0}$ is of the form $\sigma^{2} I$ where $\sigma^{2}>0$ and $I$ is the identity matrix.

A survey of various approximations and bounds for $\mathcal{D}_{0}$ can be found in [15] Chapters $5-7$ and [16] Chapters 6-7 for this model and for related ones.

The approximation of $\mathcal{D}_{0}$ by periodic environments is considered in [5,6,22]. Given an environment $\omega \in \Omega$ and an integer $N>1$, construct an environment $N$-periodic on $\mathbb{Z}^{d}, d \geq 1$, by setting

$$
\dot{a}_{N}(x, y, \omega)=a(\dot{x}, \dot{y}, \omega), \quad x \sim y
$$

where $\dot{x}, \dot{y} \in \llbracket 0, N \rrbracket^{d}, \dot{x} \sim \dot{y}$ and $\dot{x} \equiv x, \dot{y} \equiv y \bmod N$ coordinatewise.

Then consider the reversible random walk on $\mathbb{Z}^{d}$ with transition probabilities given by

$$
\dot{p}_{N}(x, y, \omega)=\dot{a}_{N}(x, y, \omega) / \dot{a}_{N}(x, \omega), \quad x \sim y
$$

where $\dot{a}_{N}(x, \omega)=\sum_{y \sim x} \dot{a}_{N}(x, y, \omega)$. These induce a probability $\dot{P}_{z, N, \omega}$ on the paths starting at $z \in \mathbb{Z}^{d}$.

For $\mathbb{P}$ almost all environments, under $\dot{P}_{0, N, \omega}, n^{-1 / 2} X_{[\cdot n]}$ converges in law to a Brownian motion. Denote by $\dot{\mathcal{D}}_{N}(\omega)$ its covariance matrix and denote its entries by $\dot{\mathcal{D}}_{N}^{i j}, 1 \leq i, j \leq d$. 
In view of applications to the theory of massless gradient fields on $\mathbb{Z}^{d}$, where periodized states can be used to define the slope-independent surface tension [12], Caputo and Ioffe [6] considered periodic approximations of the homogenized diffusion matrix of a symmetric random walk on $\mathbb{Z}^{d}$ with i.i.d. and uniformly elliptic jump rates. They proved that there is a.s. convergence at a rate faster than $C N^{-\nu}$ for some constants $C$ and $\nu>0$ that depend on the dimension and the ellipticity constant.

Bourgeat and Piatnitski [5], considered elliptic differential operators with stationary and uniformly elliptic coefficients. They showed the a.s. convergence of the approximations of the effective diffusion matrix by cubic samples with periodic, Dirichlet or Neumann boundary conditions. Furthermore, using results from Yurinsky [29], they showed that under a uniform mixing condition the rate of convergence is faster than $C N^{-\nu}$ for some constants $C$ and $\nu>0$.

In [22], Owhadi proved the a.s. convergence of the periodic approximations of the diffusion matrices $\mathcal{D}_{N}$ to the homogenized diffusion matrix $\mathcal{D}_{0}$ for stationary and uniformly elliptic jump rates and for elliptic operator with stationary and uniformly elliptic coefficients. As noted in [22], the a.s. convergence could also be obtained from the principle of periodic localization [16], p. 155 and the a.s. G-convergence properties of the elliptic differential operators.

Our main results are the following estimates of the rate of convergence and of the fluctuations of $D_{N}$ around its mean.

Theorem 2. Let $\left(a(e) ; e \in \mathcal{L}^{d}\right)$ be a sequence of i.i.d. uniformly elliptic conductances with ellipticity constant $\kappa$. Then there is a constant $C, 0<C<\infty$, which depends only on $d$ and $\kappa$ such that, for all $t>0$ and $N \geq 1$,

$$
\begin{array}{r}
\max _{i, j} \mathbb{P}\left(\left|\dot{\mathcal{D}}_{N}^{i j}-\mathbb{E} \dot{\mathcal{D}}_{N}^{i j}\right| \geq t N^{-\delta}\right) \leq 4 \exp (-C t) \\
\max _{i, j} \operatorname{Var}\left(\dot{\mathcal{D}}_{N}^{i j}\right) \leq 8 C^{-2} N^{-2 \delta}
\end{array}
$$

where $2 \delta=\max \{\alpha, d-4+\alpha\}$ and $\alpha>0$ is the regularity exponent which appears in the Harnack inequality (30).

In particular, for $d \geq 5$, it provides a lower bound for the exponent $\nu$ of the estimate [6] (1.1) which does not depend on the ellipticity constant. More precisely, there is $\nu>\frac{d}{4}-1$ such that for all sufficiently large $N$,

$$
\max _{i, j} \mathbb{P}\left(\left|\dot{\mathcal{D}}_{N}^{i j}-\mathbb{E} \dot{\mathcal{D}}_{N}^{i j}\right| \geq N^{-\nu}\right) \leq \exp \left(-N^{\nu}\right)
$$

Indeed, in (3), set $t=N^{\delta / 2}$ with $2 \delta=d-4+\alpha$ and take $\nu=d / 4-1+\alpha / 8<\delta / 2$.

The proof uses the method of bounded martingale differences developed by Kesten in [18] for first passage percolation models. This method also applies to other models where there is homogenization and when some regularity results are available. See also [26] from (2.17). For both questions considered above, the quantities involved are similar to first-passage times in that they can be expressed as solutions of a variational problem. It is this aspect that will be exploited.

This method also applies to the spectral gap of a random walk in cubes in $\mathbb{Z}^{d}, d \geq 3$, with Dirichlet boundary conditions. In this situation, the regularity estimates are provided by the De Georgii-Nash-Moser theory.

The paper is organized as follows. In Section 2, the martingale estimates of [18] are given in the form that they will be used here. Theorem 1 and its extension to non uniformly elliptic conductances are proved in Section 3. The regularity estimate used for the effective conductance is simply a maximum principle. For periodic approximations of the diffusion matrices, more prerequisites are needed. They are gathered in Sections 4.1 and 4.2. Section 4 ends with the proof of Theorem 2. Tail estimates for the Dirichlet eigenvalues are given in the last section. 


\section{Martingale estimates And notations}

When the conductances are assumed to be uniformly elliptic, a stronger version of Kesten's martingale inequality can be used. The same proof applies with some simplifications. In particular, [18] Step (iii) is not needed. However the full generality of Kesten's martingale inequalities will be used when we consider conductances that are bounded above but not necessarily uniformly elliptic. They are given in the second version.

\section{Martingale estimates I.}

Let $\left(M_{n} ; n \geq 0\right)$ be a martingale with respect to the filtration $\left(\mathcal{F}_{n} ; n \geq 0\right)$.

Let $\Delta_{k}=M_{k}-M_{k-1}, k \geq 1$. If there are positive random variables $\bar{U}_{k}$ (not necessarily $\mathcal{F}_{k}$-measurable) such that for some constant $B_{0}<\infty$

$$
\mathbb{E}\left(\Delta_{k}^{2} \mid \mathcal{F}_{k-1}\right) \leq \mathbb{E}\left(U_{k} \mid \mathcal{F}_{k-1}\right) \text { for all } k \geq 1 \quad \text { and } \quad \sum_{1}^{\infty} U_{k} \leq B_{0}
$$

then $M_{n} \rightarrow M_{\infty}$ in $L^{2}$ and a.s., and $\mathbb{E}\left|M_{\infty}-M_{0}\right|^{2} \leq B_{0}$.

Moreover, if there is a constant $B_{1}<\infty$ such that for all $k \geq 1$,

$$
\left|\Delta_{k}\right| \leq B_{1}
$$

then for all $t>0$,

where $B=\max \left\{B_{0}, e B_{1}^{2}\right\}$.

$$
\mathbb{P}\left(\left|M_{\infty}-M_{0}\right|>t\right) \leq 4 \exp \left(-\frac{t}{4 \sqrt{B}}\right)
$$

\section{Martingale estimates II.}

Let $\left(M_{n} ; n \geq 0\right)$ be a martingale with respect to the filtration $\left(\mathcal{F}_{n} ; n \geq 0\right)$.

Let $\Delta_{k}=M_{k}-M_{k-1}, k \geq 1$. If there is a constant $B_{1}<\infty$ such that for all $k \geq 1$,

$$
\left|\Delta_{k}\right| \leq B_{1}
$$

if for some random variables $U_{k} \geq 0$ (not necessarily $\mathcal{F}_{k}$-measurable)

$$
\mathbb{E}\left(\Delta_{k}^{2} \mid \mathcal{F}_{k-1}\right) \leq \mathbb{E}\left(U_{k} \mid \mathcal{F}_{k-1}\right) \text { for all } k \geq 1
$$

and if there are constants $0<C_{1}, C_{2}<\infty$ and $s_{0} \geq \mathrm{e}^{2} B_{1}^{2}$ such that,

$$
\mathbb{P}\left(\sum_{k} U_{k}>s\right) \leq C_{1} \exp \left(-C_{2} s^{2}\right), \text { for all } s \geq s_{0}
$$

then there are universal constants $c_{1}$ and $c_{2}$ which do not depend on $B_{1}, s_{0}, C_{1}, C_{2}$ nor on the distribution of $\left(M_{k}\right)$ and $\left(U_{k}\right)$ such that for all $s>0$,

$$
\mathbb{P}\left(\left|M_{\infty}-M_{0}\right|>s\right) \leq c_{1}\left(1+C_{1}+\frac{C_{1}}{s_{0}^{2} C_{2}}\right) \exp \left(-c_{2} \frac{s}{s_{0}^{1 / 2}+\left(s /\left(s_{0} C_{2}\right)\right)^{1 / 3}}\right)
$$

We end this section with some notations that will be used throughout this article. On $\mathbb{R}^{d}, d \geq 1$, the $\ell_{1}$-distance, the Euclidean distance and the $\ell_{\infty}$-distance will respectively be denoted by $|\cdot|_{1},|\cdot|$ and $|\cdot|_{\infty}$. For $u: \mathbb{Z}^{d} \rightarrow \mathbb{R}^{d}$, let $\|u\|_{\infty}=\sup _{x \in \mathbb{Z}^{d}}|u(x)|_{\infty}$. 
$\eta \in \mathbb{R}^{d}$ will be considered as a column vector and its transpose will be denoted by $\eta^{\prime}$ so that $\eta^{2}=\operatorname{tr}\left(\eta \eta^{\prime}\right)$.

For $m<n \in \mathbb{N}, \llbracket m, n \rrbracket=[m, n] \cap \mathbb{N}$. With this notation, for an integer $N \geq 1, Q_{N}:=\llbracket 1, N \rrbracket^{d}, \bar{Q}_{N}:=$ $\llbracket 0, N+1 \rrbracket^{d}$ and its boundary is $\partial Q_{N}:=\bar{Q}_{N} \backslash Q_{N}$.

For $u: \bar{Q}_{N} \rightarrow \mathbb{R}$ and $x \in Q_{N}$, let $P u(x):=E_{x}\left(u\left(X_{1}\right)\right)$ and $H u(x):=u(x)-P u(x)$.

In an environment $\omega$, the conductance of an edge $e$ with endpoints $x \sim y$ is denoted by $a(e, \omega)$ or $a(x, y, \omega)$. Similarly, for a function $v$ which is defined for $x$ and $y$, the endpoints of $e$, let $v(e, \omega):=|v(x, \omega)-v(y, \omega)|$.

Because we consider conductances that are independent, identically distributed and bounded by $\kappa$, we will assume that $\mathbb{P}$ is the product measure on $\Omega=] 0, \kappa]^{\mathcal{L}^{d}}$ and that $a(e, \cdot)$ are the coordinate functions. The expectation with respect to $\mathbb{P}$ will sometimes be denoted by $\langle\cdot\rangle$ instead of $\mathbb{E}$.

Let $\left\{e_{k} ; k \geq 1\right\}$ be a fixed ordering of $\mathcal{L}^{d}$ and let $\mathcal{F}_{k}, k \geq 1$, be the $\sigma$-algebra generated by $\left\{a\left(e_{j}, \cdot\right) ; 1 \leq j \leq k\right\}$. Then for an integrable random variable $h: \Omega \rightarrow \mathbb{R}$,

$$
\mathbb{E}\left(h \mid \mathcal{F}_{k}\right)=\mathbb{E}_{\sigma} h\left([\omega, \sigma]_{k}\right)
$$

where $[\omega, \sigma]_{k} \in \Omega$ agrees with $\omega$ for the first $k$ coordinates and with $\sigma$ for all the other coordinates and $\mathbb{E}_{\sigma}$ denotes the integration with respect to $d \mathbb{P}(\sigma)$.

\section{EFFECTIVE CONDUCTANCE}

In this section, we obtain tail estimates for the effective conductance of an increasing sequence of cubes under mixed boundary conditions and an upper bound on the variances.

Consider boundary conditions which can be interpreted as maintaining a fixed potential difference between two opposite faces of $Q_{N}=\llbracket 1, N \rrbracket^{d}$ while the other faces are insulated.

To describe more precisely the boundary conditions, denote the first coordinate of $x \in \mathbb{Z}^{d}$ by $x(1)$. Then denote by $\partial^{-} Q_{N}$ and $\partial^{+} Q_{N}$ the following two opposite faces of $\bar{Q}_{N}$,

$$
\partial^{-} Q_{N}=\left\{x \in \partial Q_{N} ; x(1)=0\right\} \text { and } \partial^{+} Q_{N}=\left\{x \in \partial Q_{N} ; x(1)=N+1\right\}
$$

and by $\partial^{i n s} Q_{N}$ the set of vertices on the insulated faces,

$$
\partial^{\text {ins }} Q_{N}=\partial Q_{N} \backslash\left(\partial^{-} Q_{N} \cup \partial^{+} Q_{N}\right) .
$$

Let $\mathcal{V}_{N}$ be the set of real-valued functions on $\bar{Q}_{N}$ such that

$$
\begin{aligned}
u(x)=0 \text { if } x \in \partial^{-} Q_{N}, & u(x)=N+1 \text { if } x \in \partial^{+} Q_{N} \\
\text { and } \quad u(x)=u(y) & \text { if } x \in \partial^{i n s} Q_{N}, y \in Q_{N} \text { and } x \sim y .
\end{aligned}
$$

Assume for the moment that the conductances $\left(a(e) ; e \in \mathcal{L}^{d}\right)$ are non-random and satisfy

$$
0<a(e) \leq \kappa, \quad \text { for all edges } e .
$$

For two functions $u, v: \bar{Q}_{N} \rightarrow \mathbb{R}$, the Dirichlet form, denoted by $\mathcal{E}_{N}$, is defined by

$$
\mathcal{E}_{N}(u, v)=\sum_{x, y} a(x, y)(u(x)-u(y))(v(x)-v(y))
$$

where the sum is over all ordered pairs $\{x, y\}$ such that $x \in Q_{N}$ and $y \in \bar{Q}_{N}$.

For all $N \geq 1$, there is a unique $v_{N} \in \mathcal{V}_{N}$, called the equilibrium potential, such that

$$
H v_{N}=0 \text { on } Q_{N} .
$$


The equilibrium potential is also a solution of a variational problem : it is the unique element of $\mathcal{V}_{N}$ such that

$$
\mathcal{E}_{N}\left(v_{N}, v_{N}\right)=\inf \left\{\mathcal{E}_{N}(u, u) ; u \in \mathcal{V}_{N}\right\} .
$$

This provides a variational representation of the effective conductance between two opposite sides of the cube $\bar{Q}_{N}$ since

$$
\mathcal{C}_{N}=(N+1)^{-2} \mathcal{E}_{N}\left(v_{N}, v_{N}\right) .
$$

Finally by the maximum principle, if $u: \bar{Q}_{N} \rightarrow \mathbb{R}$ verifies $H u=0$ on $Q_{N}$ then

$$
\max _{\bar{Q}_{N}} u=\max _{\partial Q_{N}} u .
$$

Reintroduce a random media by assuming that $\left(a(e) ; e \in \mathcal{L}^{d}\right)$ is a sequence of i.i.d. random variables satistying (10) a.s.

We will write $\mathcal{E}_{N}\left(v_{N}, v_{N}\right)_{\omega}$ to indicate that both $\mathcal{E}_{N}$ and $v_{N}$ are random and are calculated with the conductances $a(e, \omega)$ given by the environment $\omega$. Then

$$
A_{N}(\omega)=(N+1)^{2} N^{-d} \mathcal{C}_{N}(\omega)=N^{-d} \mathcal{E}_{N}\left(v_{N}, v_{N}\right)_{\omega} .
$$

Since the maximum principle does not require uniform ellipticity, it is possible to obtain good estimates under weaker conditions on the conductances with these boundary conditions. Theorem 1 given in the introduction gives tail estimates for uniformly elliptic conductances while Theorem 3 given below applies when they are not.

This model was considered by Wehr [28]. He showed that for some laws $\mathbb{P}$, which include the exponential and the one-sided normal distributions, and assuming that $\mathbb{E}\left(A_{N}\right)$ is bounded below by a positive constant, then

$$
\liminf _{N} N^{d} \operatorname{Var}\left(A_{N}\right)>0 .
$$

See also the recent paper of Benjamini and Rossignol [1] Section 5.

If the conductances are uniformly elliptic and stationary, then $A_{N}$ converges $\mathbb{P}$ a.s. and in $L^{1}(\mathbb{P})$, as $N \rightarrow \infty$. This was done in [2] Section 10 by adapting the homogenization methods of [16] Chapter 7.

For conductances that are not necessarily uniformly elliptic, we have the following estimates. Additional properties of non-uniformly elliptic reversible random walks can be found in [11].

Theorem 3. Let $\left(a(e) ; e \in \mathcal{L}^{d}\right)$ be a sequence of i.i.d. conductances on $\mathbb{Z}^{d}$ such that for some constant $1 \leq \kappa<\infty, 0<a(e) \leq \kappa$ for all edges e. Let $C_{0}=64 \kappa^{3}$.

Then, for $d \geq 5, \operatorname{Var}\left(A_{N}\right) \leq 128 d \kappa^{2} N^{4-d}$ and for all $t>0$ and $N \geq 1$,

$$
\mathbb{P}\left(\left|A_{N}-\mathbb{E} A_{N}\right| \geq t N^{(4-d) / 2}\right) \leq 4 \exp (-t /(8 \kappa \sqrt{2 d})) .
$$

If moreover, for some constants $D_{0}<\infty$ and $\gamma, 0<\gamma<2$,

$$
\mathbb{P}\left(a^{-1}(e) \geq s\right) \leq D_{0} s^{1-2 / \gamma}, \quad \text { for all } s \geq 1,
$$

then, if $d \geq 4$ or if $d=3$ and $1 / 2 \leq \gamma<1$,

$$
\operatorname{Var} A_{N} \leq 16 C_{0}\left(D_{0}+1\right) N^{2-d+\gamma}
$$

and for all $0<t<\left(\frac{C_{0}\left(D_{0}+1\right)^{5}}{8}\right)^{1 / 2} N^{(d-6+5 \gamma) / 2}$,

$$
\mathbb{P}\left(\left|A_{N}-\mathbb{E} A_{N}\right|>t\right) \leq 11 c_{1} \exp \left(-\frac{c_{2}}{2 \sqrt{2 C_{0}\left(D_{0}+1\right)}} N^{(d-2-\gamma) / 2} t\right) .
$$


where $c_{1}$ and $c_{2}$ are the constants that appear in the martingale estimates II. In particular, they do not depend on $\kappa, d$ or $N$.

$$
\text { For } d=3 \text { and } 0<\gamma \leq 1 / 2 \text {, Var } A_{N} \leq 16 C_{0}\left(D_{0}+1\right) N^{-1 / 2} \text {. }
$$

Lemma 1. Let $\omega$ and $\sigma$ be two environments such that $a(e, \omega)=a(e, \sigma)$ for all edges except maybe for $e=e_{k}$. Then for all $N \geq 1$,

$$
\left|A_{N}(\omega)-A_{N}(\sigma)\right| \leq \kappa N^{-d}\left(v_{N}^{2}\left(e_{k}, \omega\right)+v_{N}^{2}\left(e_{k}, \sigma\right)\right)
$$

Proof. Since $v_{N} \in \mathcal{V}_{N}$ is the solution of a variational problem,

$$
\begin{aligned}
A_{N}(\omega)-A_{N}(\sigma) & =N^{-d}\left(\mathcal{E}_{N}\left(v_{N}, v_{N}\right)_{\omega}-\mathcal{E}_{N}\left(v_{N}, v_{N}\right)_{\sigma}\right) \\
& \leq N^{-d} \sum_{e}(a(e, \omega)-a(e, \sigma)) v_{N}^{2}(e, \sigma) \leq \kappa N^{-d} v_{N}^{2}\left(e_{k}, \sigma\right) .
\end{aligned}
$$

Proof of Theorem 1. With the notations of Section 2, let $\Delta_{k}=\mathbb{E}\left(A_{N} \mid \mathcal{F}_{k}\right)-\mathbb{E}\left(A_{N} \mid \mathcal{F}_{k-1}\right), M_{0}=0$ and $M_{n}=\sum_{1}^{n} \Delta_{k}, n \geq 1$.

To check that $\left(M_{n} ; n \geq 0\right)$ is a martingale that verifies conditions (5) and (6), we have by Lemma 1 ,

$$
\begin{aligned}
\left|\Delta_{k}\right| & \leq \mathbb{E}_{\sigma}\left|A_{N}\left([\omega, \sigma]_{k}\right)-A_{N}\left([\omega, \sigma]_{k-1}\right)\right| \\
& \leq \kappa N^{-d} \mathbb{E}_{\sigma} v_{N}^{2}\left(e_{k},[\omega, \sigma]_{k-1}\right)+\kappa N^{-d} \mathbb{E}_{\sigma} v_{N}^{2}\left(e_{k},[\omega, \sigma]_{k}\right) \\
& \leq 8 \kappa N^{2-d}
\end{aligned}
$$

since by the maximum principle, $0 \leq v_{N}(x) \leq N+1$, for all $x \in \bar{Q}_{N}$.

Then,

$$
\begin{aligned}
\Delta_{k}^{2} & \leq \mathbb{E}_{\sigma}\left(\left|A_{N}\left([\omega, \sigma]_{k}\right)-A_{N}\left([\omega, \sigma]_{k-1}\right)\right|^{2}\right) \\
& \leq 2 \kappa^{2} N^{-2 d} \mathbb{E}_{\sigma} v_{N}^{4}\left(e_{k},[\omega, \sigma]_{k-1}\right)+2 \kappa^{2} N^{-2 d} \mathbb{E}_{\sigma} v_{N}^{4}\left(e_{k},[\omega, \sigma]_{k}\right) \\
& \quad \text { and by the maximum principle, } \\
& \leq 8 \kappa^{2} N^{2-2 d} \mathbb{E}_{\sigma} v_{N}^{2}\left(e_{k},[\omega, \sigma]_{k-1}\right)+8 \kappa^{2} N^{2-2 d} \mathbb{E}_{\sigma} v_{N}^{2}\left(e_{k},[\omega, \sigma]_{k}\right) .
\end{aligned}
$$

For $k \geq 1$, let

We see that $\mathbb{E}\left(\Delta_{k}^{2} \mid \mathcal{F}_{k-1}\right) \leq \mathbb{E}\left(U_{k} \mid \mathcal{F}_{k-1}\right)$.

$$
U_{k}(\omega)=16 \kappa^{2} N^{2-2 d} v_{N}^{2}\left(e_{k}, \omega\right) .
$$

By (1) and (12), $\mathcal{E}_{N}\left(v_{N}, v_{N}\right)_{\omega} \leq 2 \kappa N^{d}$. Then by uniform ellipticity,

$$
\begin{aligned}
\sum_{k} U_{k} & =16 \kappa^{2} N^{2-2 d} \sum_{k} v_{N}^{2}\left(e_{k}, \omega\right) \\
& \leq 16 \kappa^{3} N^{2-2 d} \mathcal{E}_{N}\left(v_{N}, v_{N}\right)_{\omega}<16 \kappa^{3}(2 \kappa) N^{2-2 d+d}
\end{aligned}
$$

Hence condition (5) holds with $B_{0}=64 \kappa^{4} N^{2-d}$ and condition (6) holds with $B_{1}=4 \kappa N^{2-d}$. Therefore, $A_{N}-\mathbb{E} A_{N}=\sum_{1}^{\infty} \Delta_{k}$ a.s. and in $L^{2}$ and for $d \geq 3$, by the martingale estimates I, we have that for all $N \geq 1$ and $t \geq 0$,

$$
\mathbb{P}\left(\left|A_{N}-\mathbb{E} A_{N}\right| \geq t\right) \leq 4 \exp \left(-\frac{t}{4 \sqrt{B}}\right)
$$

where $B=\max \left\{B_{0}, e B_{1}^{2}\right\}=64 \kappa^{4} N^{2-d}$. Accordingly, $\operatorname{Var}\left(A_{N}\right) \leq 64 \kappa^{4} N^{2-d}$. 
Proof of Theorem 3. To obtain (13), the preceding proof can be used up to (16) where uniform ellipticity is first needed.

Let $U_{k}(\omega)=16 \kappa^{2} N^{2-2 d} v_{N}^{2}\left(e_{k}, \omega\right), k \geq 1$.

Then simply bound $v_{N}^{2}\left(e_{k}, \omega\right)$ by $4 N^{2}$ to obtain that

$$
\sum_{k} U_{k} \leq 128 d \kappa^{2} N^{4-d}
$$

Hence the martingale estimates I hold with $B_{0}=128 d \kappa^{2} N^{4-d}, B_{1}=4 \kappa N^{2-d}$ and $B=\max \left\{B_{0}, e B_{1}^{2}\right\}=B_{0}$.

These estimates can be improved if we assume that (14) holds for some $0<\gamma<2$. Starting from (16), we have that for all $N \geq 1$,

$$
\begin{aligned}
\sum_{k} v_{N}^{2}\left(e_{k}, \omega\right) & \leq N^{\gamma} \sum_{k} a\left(e_{k}, \omega\right) v_{N}^{2}\left(e_{k}, \omega\right)+4 N^{2} \sharp\left\{k ; a\left(e_{k}, \omega\right) \leq N^{-\gamma}\right\} \\
& \leq 2 \kappa N^{d+\gamma}+4 N^{2} \sharp\left\{k ; a\left(e_{k}, \omega\right) \leq N^{-\gamma}\right\} .
\end{aligned}
$$

Therefore, $\sum_{k} U_{k} \leq 32 \kappa^{3} N^{2-d+\gamma}+64 \kappa^{2} N^{4-2 d_{\sharp}} \sharp\left\{k ; a\left(e_{k}, \omega\right) \leq N^{-\gamma}\right\}$.

Let $C_{0}=64 \kappa^{3}$. Then for $t>1, N \geq 1$ and $\gamma>0$,

$$
\begin{aligned}
\mathbb{P}\left(\sum_{k} U_{k}>C_{0} t N^{2-d+\gamma}\right) & \leq \mathbb{P}\left(\sharp\left\{k ; a\left(e_{k}, \omega\right) \leq N^{-\gamma}\right\}>(t-1) N^{d-2+\gamma}\right) \\
& \leq 2 \exp \left(-\frac{N^{d}}{4}\left((t-1) N^{\gamma-2}-p_{N}\right)^{2}\right)
\end{aligned}
$$

by Bernstein's inequality with $p_{N}=\mathbb{P}\left(a^{-1}(e)>N^{\gamma}\right)$. By (14), we have that for all $N \geq 1, p_{N} \leq D_{0} N^{\gamma-2}$. Therefore, if $t>2\left(1+D_{0}\right)$ then $\left(t-1-D_{0}\right)^{2}>t^{2} / 4$ and

$$
\begin{aligned}
\mathbb{P}\left(\sum_{k} U_{k}>C_{0} t N^{2-d+\gamma}\right) & \leq 2 \exp \left(-\frac{N^{d}}{4}\left(t-1-D_{0}\right)^{2} N^{2 \gamma-4}\right) \\
& \leq 2 \exp \left(-\frac{t^{2}}{16} N^{d-4+2 \gamma}\right)
\end{aligned}
$$

Equivalently, for all $E s \geq 2 C_{0}\left(D_{0}+1\right) N^{2-d+\gamma}$,

$$
\mathbb{P}\left(\sum_{k} U_{k}>s\right) \leq 2 \exp \left(-\frac{s^{2}}{16 C_{0}^{2}} N^{3 d-8}\right)
$$

We see that the conditions for the martingale estimates II hold with

$$
B_{1}=4 \kappa N^{2-d}, C_{1}=2, C_{2}=\frac{1}{16 C_{0}^{2}} N^{3 d-8} \text { and } s_{0}=2 C_{0}\left(D_{0}+1\right) N^{2-d+\gamma}
$$

since $C_{0} \geq 8 \mathrm{e}^{2} \kappa^{2}$. 
In particular, we have the tail estimates (15).

Moreover,

$$
\begin{aligned}
\operatorname{Var} A_{N} & \leq \mathbb{E} \sum_{k} U_{k}=\int_{0}^{\infty} \mathbb{P}\left(\sum_{k} U_{k}>s\right) \mathrm{d} s \\
& \leq s_{0}+\int_{s_{0}}^{\infty} C_{1} \mathrm{e}^{-C_{2} s^{2}} \mathrm{~d} s .
\end{aligned}
$$

Hence, if $2-d+\gamma \geq 6-2 d-\gamma$,

$$
\operatorname{Var} A_{N} \leq s_{0}+\frac{C_{1}}{s_{0} C_{2}} \leq 16 C_{0}\left(D_{0}+1\right) N^{2-d+\gamma}
$$

while for $d=3$ and $\gamma-1 \leq-1 / 2$,

$$
\operatorname{Var} A_{N} \leq s_{0}+\frac{C_{1}}{\sqrt{C}_{2}} \leq 16 C_{0}\left(D_{0}+1\right) N^{-1 / 2} .
$$

\section{THE PERIODiC APPROXIMATION}

The first step of the proof will be to express the covariance matrices $\dot{\mathcal{D}}_{N}$ in terms of periodic corrector fields. The second step will be to obtain regularity estimates for the corrector fields. The proof will then be completed with the martingale estimates.

The expectation with respect to $\dot{P}_{0, N, \omega}$ will be denoted by $\dot{E}_{z, N, \omega}$. We will also use the Laplacian $\dot{H}_{N, \omega}$ which is defined for a function $u: \mathbb{Z}^{d} \rightarrow \mathbb{R}$ by $\dot{H}_{N, \omega} u(x)=u(x)-\dot{E}_{x, N, \omega} u\left(X_{1}\right)$.

\subsection{Periodic corrector fields}

A periodic corrector field for the random walk $\left(X_{n} ; n \geq 0\right)$ in the periodic environment is a function $\dot{\chi}_{N}$ : $\mathbb{Z}^{d} \times \Omega \rightarrow \mathbb{R}^{d}$ with the property that $\mathbb{P}$-a.s.

$$
X_{n}+\dot{\chi}_{N}\left(X_{n}\right), \quad n \geq 0
$$

is a martingale with respect to $\dot{P}_{0, N, \omega}$.

Therefore, $\mathbb{P}$-a.s., $\dot{\chi}_{N}$ must verify the equations $\dot{E}_{x, N}\left(X_{1}+\dot{\chi}_{N}\left(X_{1}\right)\right)=x+\dot{\chi}_{N}(x)$ for all $x \in \mathbb{Z}^{d}$, or equivalently,

$$
\dot{H}_{N} \dot{\chi}_{N}(x)=\dot{d}_{N}(x), \quad \text { for all } x \in \mathbb{Z}^{d},
$$

where $\dot{d}_{N}(x)=\dot{E}_{x, N}\left(X_{1}\right)-x$ is the drift of the walk. Note that each coordinate of $\dot{d}_{N}$ is $N$-periodic.

The vector space of $N$-periodic functions on $\mathbb{Z}^{d}$ can be identified with

$$
\dot{\mathcal{H}}_{N}=\left\{u: \bar{Q}_{N} \rightarrow \mathbb{R} ; u(x)=u(y) \forall x, y \in \bar{Q}_{N} \text { such that } x \equiv y \bmod N\right\} .
$$

Let $u \in \dot{\mathcal{H}}_{N}$. By considering $u$ as an $N$-periodic function on $\mathbb{Z}^{d}, \dot{H}_{N} u(x)$ is defined for all $x \in \mathbb{Z}^{d}$. Furthermore, since $\dot{H}_{N} u$ is $N$-periodic on $\mathbb{Z}^{d}$, its restriction to $\bar{Q}_{N}$ belongs to $\dot{\mathcal{H}}_{N}$. This procedure defines a bounded linear operator $\dot{H}_{N}: \dot{\mathcal{H}}_{N} \rightarrow \dot{\mathcal{H}}_{N}$.

For two functions $u, v: \bar{Q}_{N} \rightarrow \mathbb{R}$, define the norm, the scalar product, and the Dirichlet form respectively by $\|u\|_{p, \dot{N}}^{p}=\sum_{x \in Q_{N}}|u(x)|^{p} \dot{a}_{N}(x), 1 \leq p<\infty,(u, v)_{\dot{N}}=\sum_{x \in Q_{N}} u(x) v(x) \dot{a}_{N}(x)$ and

$$
\dot{\mathcal{E}}_{N}(u, v)=\sum_{x, y} \dot{a}_{N}(x, y)(u(x)-u(y))(v(x)-v(y))
$$


where the sum is over all ordered pairs $\{x, y\}$ such that $x \in Q_{N}$ and $y \in \llbracket 0, N \rrbracket^{d}$. This expression makes sense for all functions $u, v: \mathbb{Z}^{d} \rightarrow \mathbb{R}$. But if both $u, v$ are $N$-periodic, then the Green-Gauss formula holds

$$
\dot{\mathcal{E}}_{N}(u, v)=\left(u, \dot{H}_{N} v\right)_{\dot{N}}
$$

Let $\dot{\mathcal{H}}_{N}^{0}(\omega)=\left\{u \in \dot{\mathcal{H}}_{N} ;(u, 1)_{\dot{N}}=0\right\}$. Note that $\dot{\mathcal{H}}_{N}^{0}$ depends on the environment but $\dot{\mathcal{H}}_{N}$ does not.

All the properties of the solutions of a Poisson equation that will be needed are gathered in the following proposition.

Proposition 1. Let $\left(a(e) ; e \in \mathcal{L}^{d}\right), d \geq 1$, be a stationary sequence of uniformly elliptic conductances. Then IP-a.s.,

(1) $\dot{H}_{N}: \dot{\mathcal{H}}_{N}^{0} \rightarrow \dot{\mathcal{H}}_{N}^{0}$ is a bounded invertible linear operator.

(2) For $f: Q_{N} \rightarrow \mathbb{R}, \dot{H}_{N} u=f$ possesses a unique solution $u \in \dot{\mathcal{H}}_{N}$ if and only if $f \in \dot{\mathcal{H}}_{N}^{0}$.

(3) Let $f \in \dot{\mathcal{H}}_{N}^{0}$.

(a) The infimum $\inf _{\dot{\mathcal{H}}_{N}^{0}}\left[\dot{\mathcal{E}}_{N}(u, u)-2(u, f)\right]$ is attained by the solution $u \in \dot{\mathcal{H}}_{N}^{0}$ of the equation $\dot{H}_{N} u=f$.

(b) The infimum $\gamma:=\inf _{\mathcal{M}} \dot{\mathcal{E}}_{N}(u, u)$ where $\mathcal{M}=\left\{u \in \dot{\mathcal{H}}_{N}^{0} ; E(f, u)_{\dot{N}}=1\right\}$ is attained by the solution $u \in \dot{\mathcal{H}}_{N}^{0}$ of the equation $\dot{H}_{N} u=\gamma f$.

(4) If $f \in \dot{\mathcal{H}}_{N}^{0}$ then the unique solution $u \in \dot{\mathcal{H}}_{N}^{0}$ of $\dot{H}_{N} u=f$ is

$$
u=\int_{0}^{\infty} \mathrm{e}^{-t \dot{H}_{N}} f \mathrm{~d} t, \quad x \in Q_{N} .
$$

(5) There is a constant $C=C(d, \kappa)<\infty$ such that for all $N \geq 1$ and $f \in \dot{\mathcal{H}}_{N}^{0}, u \in \dot{\mathcal{H}}_{N}^{0}$, the solution of $\dot{H}_{N} u=f$, verifies the regularity estimates,

$$
\|u\|_{\infty} \leq C N^{2}\|f\|_{2, \dot{N}} \quad \text { and } \quad\|u\|_{\infty} \leq C N^{2}(\log N)\|f\|_{\infty} .
$$

Proof. For all $u \in \dot{\mathcal{H}}_{N}^{0}, \dot{H}_{N} u \in \dot{\mathcal{H}}_{N}^{0}$ by the Green-Gauss formula (18).

$\dot{H}_{N}: \dot{\mathcal{H}}_{N}^{0} \rightarrow \dot{\mathcal{H}}_{N}^{0}$ is invertible since if $\dot{H}_{N} u=0$ then $u$ is constant by the maximum principle.

The variational principle $3 b$ holds for the Poisson equation on a smooth compact Riemannian manifold with $f \in \mathcal{C}^{\infty}$, see [14] Proposition 2.6 due to Druet. The same arguments can be used.

Suppose that $f$ is not identically 0 . Then $\mathcal{M}$ is a closed convex set which is not empty since $\|f\|_{2, N}^{-2} f \in \mathcal{M}$. Therefore the infimum, $\gamma$, is attained for some $u_{0} \in \mathcal{M}$ and $\gamma>0$ since $u_{0}$ is not constant. Then using a theorem by Lagrange, there are two constants $\alpha, \beta \in \mathbb{R}$ such that for all $x \in Q_{N}$,

$$
2 \sum_{y \sim x}\left(u_{0}(x)-u_{0}(y)\right) \dot{a}_{N}(x, y)-\alpha f(x) \dot{a}_{N}(x)-\beta \dot{a}_{N}(x)=0
$$

and $2 \dot{H}_{N} u_{0}(x)-\alpha f(x)-\beta=0$. Therefore, for all $\varphi \in \dot{\mathcal{H}}_{N}$,

$$
2 \dot{\mathcal{E}}_{N}\left(u_{0}, \varphi\right)=\alpha(f, \varphi)_{\dot{N}}+\beta(1, \varphi)_{\dot{N}}
$$

For $\varphi=1$, one finds that $\beta=0$ while for $\varphi=u_{0}$, one finds that $\alpha=2 \gamma$. Hence $\left(H u_{0}, \varphi\right)_{\dot{N}}=\gamma(f, \varphi)_{\dot{N}}$ for all $\varphi \in \dot{\mathcal{H}}_{N}$.

The variational principle $3 a$ can be proven similarly.

To prove the last two properties, the estimate of the speed of convergence to equilibrium of a Markov chain on a finite state space given in terms of the spectral gap is needed. A more detailed survey is given in [25] Section 2.1 
Let $\dot{K}_{N}(t, x, y), t \geq 0$ and $x, y \in Q_{N}$, be the heat kernel of $\mathrm{e}^{-t \dot{H}_{N}}$. Then for all $t \geq 0$ and $x, y \in Q_{N}$, $\dot{K}_{N}(t, x, y) \geq 0$ and $E \sum_{y} \dot{K}_{N}(t, x, y)=1$. In particular, for all $f \in \dot{\mathcal{H}}_{N}$ and $t \geq 0$,

$$
\left\|\mathrm{e}^{-t \dot{H}_{N}} f\right\|_{\infty} \leq\|f\|_{\infty} .
$$

Denote the volume of the torus $Q_{N}$, the invariant probability for the random walk on $Q_{N}$ and the smallest non zero eigenvalue of $\dot{H}_{N}$ on $\dot{\mathcal{H}}_{N}$ respectively by

$$
\dot{a}_{N}\left(Q_{N}\right)=\sum_{x \in Q_{N}} \dot{a}_{N}(x), \quad \dot{\pi}_{N}(x)=\dot{a}_{N}(x) / \dot{a}_{N}\left(Q_{N}\right) \quad \text { and } \quad \dot{\lambda}_{N} .
$$

With these notations, we have the following very useful inequality. A proof can be found in [25] p. 328 for instance. For all $t>0$ and $x, y \in Q_{N}$,

$$
\left|\dot{K}_{N}(t, x, y)-\dot{\pi}_{N}(y)\right| \leq \kappa \exp \left(-t \dot{\lambda}_{N}\right) .
$$

Therefore, for all $t>0$ and $f \in \dot{H}_{N}^{0}$,

$$
\left\|\mathrm{e}^{-t \dot{H}_{N}} f\right\|_{\infty}=\sup _{x}\left|\sum_{y}\left(\dot{K}_{N}(t, x, y)-\dot{\pi}_{N}(y)\right) f(y)\right| \leq \kappa \exp \left(-t \dot{\lambda}_{N}\right)\|f\|_{1, \dot{N}} .
$$

By the Riesz-Thorin interpolation theorem, from (19) and (21), we obtain that

$$
\left\|\mathrm{e}^{-t \dot{H}_{N}} f\right\|_{\infty} \leq \sqrt{\kappa} \exp \left(-t \dot{\lambda}_{N} / 2\right)\|f\|_{2, \dot{N}} .
$$

This will be completed by the following lower bound on $\dot{\lambda}_{N}$. There exists a constant $C_{1}>0$ which depends only on the dimension and on the ellipticity constant $\kappa$ such that $\mathbb{P}$ a.s. and for all $N \geq 1$,

$$
N^{2} \dot{\lambda}_{N}>C_{1} \text {. }
$$

This follows from the Courant-Fischer min-max principle [25], p. 319 by comparison with the eigenvalues of the simple symmetric random walk which corresponds to the case where the conductance of every edge is 1 . For Neumann boundary conditions the expressions are not as explicit but for Dirichlet and periodic boundary conditions on $Q_{N}$, the eigenvalues can be calculated explicitely much as in [27]. We find that for each $\xi \in \llbracket 0, N \llbracket^{d}$, there is an eigenvalue for the periodic boundary conditions on $Q_{N}, \lambda_{\xi}\left(Q_{N}\right)$, that verifies

$$
\lim _{N \rightarrow \infty} N^{2} \lambda_{\xi}\left(Q_{N}\right)=\frac{\pi^{2}}{d} \sum_{|z|=1}(\xi \cdot z)^{2} \quad \text { as } \quad N \rightarrow \infty .
$$

The representation formula given in 4 follows from the spectral estimates (20) and (23). See [23] for another recent application of 4 .

The first regularity result follows from the representation formula (4) and (22): for $f \in \dot{\mathcal{H}}_{N}^{0}$,

$$
\|u\|_{\infty} \leq \int_{0}^{\infty}\left\|\mathrm{e}^{-s \dot{H}_{N}} f\right\|_{\infty} \mathrm{d} s \leq \int_{0}^{\infty} \sqrt{\kappa} \mathrm{e}^{-s \dot{\lambda}_{N} / 2}\|f\|_{2, \dot{N}} \mathrm{~d} s \leq C N^{2}\|f\|_{2, \dot{N}} .
$$

The second one follows from (19) and (21) : for $f \in \dot{\mathcal{H}}_{N}^{0}$ and $t>0$,

$$
\|u\|_{\infty} \leq \int_{0}^{t}\left\|\mathrm{e}^{-s \dot{H}_{N}} f\right\|_{\infty} \mathrm{d} s+\int_{t}^{\infty}\left\|\mathrm{e}^{-s \dot{H}_{N}} f\right\|_{\infty} \mathrm{d} s \leq t\|f\|_{\infty}+\frac{\mathrm{e}^{-t \dot{\lambda}_{N}}}{\dot{\lambda}_{N}}\|f\|_{1, \dot{N}} .
$$


Use $\|f\|_{1, \dot{N}} \leq 2 \mathrm{~d} \kappa N^{d}\|f\|_{\infty}$ and let $t=\frac{d}{C_{1}} N^{2} \log N$.

For a function $u: \bar{Q}_{N} \rightarrow \mathbb{R}^{d}$, define $\dot{H}_{N} u$ in $Q_{N}$ by applying it coordinatewise.

Let $g: \mathbb{Z}^{d} \rightarrow \mathbb{Z}^{d}$ be the function defined by $g(x)=x$.

Corollary 1. Let $\left(a(e) ; e \in \mathcal{L}^{d}\right), d \geq 1$, be a stationary sequence of uniformly elliptic conductances. Then for all $N \geq 1$, there is a unique function $\dot{\chi}_{N}: \bar{Q}_{N} \times \Omega \rightarrow \mathbb{R}^{d}$ such that $\mathbb{P}$-a.s., in each coordinate, it is in $\dot{\mathcal{H}}_{N}^{0}(\omega)$ and

$$
\dot{H}_{N} \dot{\chi}_{N}=-\dot{H}_{N} g, \quad \text { on } Q_{N}
$$

Moreover, there is a constant $C=C(d, \kappa)$ such that

$$
\left\|\dot{\chi}_{N}\right\|_{\infty} \leq C N^{2} \log N
$$

Proof. Note that for each coordinate of $-\dot{H}_{N} g$ belongs to $\dot{\mathcal{H}}_{N}^{0}$ :

Indeed $\dot{H}_{N} g$ is $N$-periodic and

$$
\begin{aligned}
\sum_{x \in Q_{N}} \dot{H}_{N} g(x) \dot{a}_{N}(x) & =\sum_{x} \sum_{y \sim x} \dot{a}_{N}(x) \dot{p}_{N}(x, y)(g(x)-g(y)) \\
& =\sum_{x} \sum_{y \sim x} \dot{a}_{N}(x, y)(x-y)=0 .
\end{aligned}
$$

Then use property 5 of proposition 1 with the function $f=-\dot{H}_{N} g$. The regularity estimate (25) follows since $\|f\|_{\infty} \leq 1$.

The next step is to express the covariance matrix of the walk in a periodic environment in terms of $\dot{\chi}_{N}$. By (17), $M_{n}=X_{n}+\dot{\chi}_{N}\left(X_{n}\right), n \geq 0$ is a martingale with uniformly bounded increments: $Z_{n}=M_{n}-M_{n-1}$.

Let $h(x)=\dot{E}_{x, N}\left(Z_{1} Z_{1}^{\prime}\right)$. Since $h \in \dot{\mathcal{H}}_{N}$, by the ergodic theorem for a Markov chain on $Q_{N}, \dot{P}_{0, N}$ a.s.,

$$
\frac{1}{n} \sum_{1}^{n} \dot{E}_{0, N}\left(Z_{j} Z_{j}^{\prime} \mid X_{j-1}\right)=\frac{1}{n} \sum_{1}^{n} h\left(X_{j-1}\right) \rightarrow \sum_{Q_{N}} \dot{\pi}_{N}(x) h(x) \text { as } n \rightarrow \infty .
$$

Then by the martingale central limit theorem (see [10], (7.4) Chap. 7), $\frac{1}{\sqrt{n}} M_{n}$ converges to a Gaussian law. Hence $\frac{1}{\sqrt{n}} X_{n}$ also converges to a Gaussian with the same covariance matrix which is given by

$$
\begin{aligned}
\dot{\mathcal{D}}_{N} & =\sum_{Q_{N}} \dot{\pi}_{N}(x) h(x) \\
& =\dot{a}_{N}\left(Q_{N}\right)^{-1} \sum_{x \sim y} \dot{a}_{N}(x, y)\left(\dot{v}_{N}(y)-\dot{v}_{N}(x)\right)\left(\dot{v}_{N}(y)-\dot{v}_{N}(x)\right)^{\prime} .
\end{aligned}
$$

where $\dot{v}_{N}(x)=x+\dot{\chi}_{N}(x)$. 
For uniformly elliptic, stationary and ergodic conductances, the effective diffusion matrix of the jump process in a periodic environment converges to the homogenized effective diffusion matrix $\mathcal{D}_{0}$ (see [5] Th. 1 and [22] Th. 4.1). And since the jump process and the random walk on $\mathbb{Z}^{d}$ have the same diffusion matrix, we have that $\dot{\mathcal{D}}_{N} \rightarrow \mathcal{D}_{0} \mathbb{P}$-a.s. as $N \rightarrow \infty$.

To write $\dot{\mathcal{D}}_{N}$ in terms of the Dirichlet form on $\dot{\mathcal{H}}_{N}$, we will extend the definition of $\dot{\mathcal{E}}_{N}$ to $\mathbb{R}^{d}$-valued functions so that the expression of $\dot{\mathcal{D}}_{N}$ given in (26) becomes

$$
\dot{\mathcal{D}}_{N}=\dot{a}_{N}\left(Q_{N}\right)^{-1} \dot{\mathcal{E}}_{N}\left(\dot{v}_{N}, \dot{v}_{N}\right)
$$

where $\dot{v}_{N}=g+\dot{\chi}_{N}$.

For two functions $u, v: \bar{Q}_{N} \rightarrow \mathbb{R}^{d}$, define

$$
\begin{aligned}
(u, v)_{\dot{N}} & =\sum_{x \in Q_{N}} u(x) v(x)^{\prime} \dot{a}_{N}(x), \\
\text { and } \quad \dot{\mathcal{E}}_{N}(u, v) & =\sum_{x, y} \dot{a}_{N}(x, y)(u(x)-u(y))(v(x)-v(y))^{\prime}
\end{aligned}
$$

where the sum is over all ordered pairs $\{x, y\}$ such that $x \in Q_{N}$ and $y \in \llbracket 0, N \rrbracket^{d}$.

Coordinatewise, the periodic corrector fields are the solutions of variational problems. Indeed, from the variational formula $3 a$ of proposition 1 , we have that $\mathbb{P}$ a.-s. and for all $N \geq 1$,

$$
\begin{aligned}
\inf \left\{\operatorname{tr} \dot{\mathcal{E}}_{N}(g+u, g+u) ; u \in\left(\dot{\mathcal{H}}_{N}^{0}\right)^{d}\right\} & =\operatorname{tr} \dot{\mathcal{E}}_{N}(g, g)+\inf \left\{\operatorname{tr} \dot{\mathcal{E}}_{N}(u, u)-2 \operatorname{tr}(f, u) ; u \in\left(\dot{\mathcal{H}}_{N}^{0}\right)^{d}\right\} \\
& =\operatorname{tr} \dot{\mathcal{E}}_{N}\left(\dot{v}_{N}, \dot{v}_{N}\right)
\end{aligned}
$$

where $g(x)=x$ and $f=-\dot{H}_{N} g$ as in Corollary 1 .

In particular, since

$$
\operatorname{tr} \dot{\mathcal{E}}_{N}\left(\dot{\chi}_{N}, \dot{\chi}_{N}\right) \leq 2 \operatorname{tr} \dot{\mathcal{E}}_{N}\left(\dot{v}_{N}, \dot{v}_{N}\right)+2 \operatorname{tr} \dot{\mathcal{E}}_{N}(g, g) \leq 4 \operatorname{tr} \dot{\mathcal{E}}_{N}(g, g),
$$

there is a constant $C=C(d, \kappa)<\infty$ such that $\mathbb{P}$-a.s. and for all $N \geq 1$,

$$
\operatorname{tr} \dot{\mathcal{E}}_{N}\left(\dot{\chi}_{N}, \dot{\chi}_{N}\right) \leq C N^{d}
$$

The second variational principle, $3 b$ of Proposition 1, could be used to obtain a lower bound on $\operatorname{tr} \dot{\mathcal{E}}_{N}\left(\chi_{N}, \chi_{N}\right)$.

\subsection{Further regularity results}

In the following proposition, we improve the estimate given in (25) for dimensions $2 \leq d \leq 4$.

Proposition 2. Let $\left(a(e) ; e \in \mathcal{L}^{d}\right), d \geq 2$, be a stationary sequence of uniformly elliptic conductances. Then there is a constant $C=C(d, \kappa)<\infty$, such that for all $N \geq 1$

$$
\left\|\dot{\chi}_{N}\right\|_{\infty} \leq\left\{\begin{array}{cc}
C N^{d / 2} & \text { for } d \geq 3 \\
C N(\log N)^{1 / 2} & \text { for } d=2
\end{array}\right.
$$

Proof. For $\eta \in \mathbb{R}^{d}$, let $z_{0}$ and $z_{1}$ be two vertices of $\mathbb{Z}^{d}$ such that

$$
\eta \cdot \dot{\chi}_{N}\left(z_{0}\right)=\min _{x \in \mathbb{Z}^{d}} \eta \cdot \dot{\chi}_{N}(x) \text { and } \eta \cdot \dot{\chi}_{N}\left(z_{1}\right)=\max _{x \in \mathbb{Z}^{d}} \eta \cdot \dot{\chi}_{N}(x)
$$

Since $\dot{\chi}_{N}$ is $N$-periodic, we can assume that $\left|z_{0}-z_{1}\right|_{\infty} \geq N$.

Let $z_{0}=x_{0}, x_{1}, \ldots, x_{n-1}, x_{n}=z_{1}$ be a path from $z_{0}$ to $z_{1}$ such that $1 \leq n \leq d N$. 


$$
\begin{aligned}
& \text { For } i=0 \text { or } 1 \text {, let } \bar{w}_{i}(y)=\left(\frac{\left|z_{1}-z_{0}\right|_{\infty}}{1+\left|y-z_{i}\right|_{\infty}}\right)^{d-1} \text { and } \\
& \qquad \mathcal{P}_{i}=\left\{y \in \mathbb{Z}^{d} ;\left|y-z_{i}\right|_{\infty} \leq \frac{1}{2}\left|z_{1}-z_{0}\right|_{\infty}\right\}
\end{aligned}
$$

For $\mathcal{C}$, a given set of finite paths in $\mathbb{Z}^{d}$, let $w(y)=\operatorname{card}\{\gamma \in \mathcal{C} ; y \in \gamma\}$.

Then by [3], Lemma 2, p. 26, there is a constant $C<\infty$, which depends only on the dimension $d$, and there is a set of paths $\mathcal{C}$ from $z_{0}$ to $z_{1}$ such that

$$
\operatorname{card} \mathcal{C}=\left|z_{1}-z_{0}\right|_{\infty}^{d-1}
$$

and such that for all $y \in \mathbb{Z}^{d}$,

$$
w(y) \leq C \bar{w}_{i}(y) \text { if } y \in \mathcal{P}_{i} \text { and } w(y)=0 \text { otherwise. }
$$

For each path of $\mathcal{C}, z_{0}=x_{0}, x_{1} \ldots, x_{n-1}, x_{n}=z_{1}$ and for all $x \in \mathbb{Z}^{d}$,

$$
\left|\eta \cdot \dot{\chi}_{N}(x)\right|_{\infty} \leq\left|\eta \cdot \dot{\chi}_{N}\left(z_{1}\right)-\eta \cdot \dot{\chi}_{N}\left(z_{0}\right)\right|_{\infty} \leq|\eta|_{\infty} \sum_{j=1}^{n}\left|\dot{\chi}_{N}\left(x_{j}\right)-\dot{\chi}_{N}\left(x_{j-1}\right)\right|_{\infty} .
$$

Since this holds for all paths in $\mathcal{C}$, it also holds for the arithmetic average over the paths of $\mathcal{C}$. Therefore,

$$
\left|\eta \cdot \dot{\chi}_{N}(x)\right|_{\infty} \leq \frac{|\eta|_{\infty}}{\left|z_{1}-z_{0}\right|_{\infty}^{d-1}} \sum_{y \in \mathcal{P}_{0} \cup \mathcal{P}_{1}} w(y) h_{N}(y)
$$

where $h_{N}(y)=\sum_{z \sim y}\left|\dot{\chi}_{N}(y)-\dot{\chi}_{N}(z)\right|_{\infty}$.

By (27) and (29),

$$
\begin{aligned}
\left|z_{1}-z_{0}\right|_{\infty}^{1-d} \sum_{y \in \mathcal{P}_{1}} w(y) h_{N}(y) & \leq C\left|z_{1}-z_{0}\right|_{\infty}^{1-d} \sum_{y \in \mathcal{P}_{1}} \bar{w}_{1}(y) h_{N}(y) \\
& \leq \frac{C}{N^{d-1}}\left(\sum_{y \in \mathcal{P}_{1}} \bar{w}_{1}^{2}(y)\right)^{1 / 2}\left(\sum_{y \in \mathcal{P}_{1}} h_{N}^{2}(y)\right)^{1 / 2} \\
& \leq \frac{C}{N^{d-1}}\left(\int_{1}^{N}\left(\frac{N}{r}\right)^{2(d-1)} r^{d-1} \mathrm{~d} r\right)^{1 / 2}\left(\operatorname{tr} \dot{\mathcal{E}}_{N}\left(\dot{\chi}_{N}, \dot{\chi}_{N}\right)\right)^{1 / 2} \\
& \leq C N^{d / 2}\left(\int_{1}^{N} r^{1-d} \mathrm{~d} r\right)^{1 / 2}
\end{aligned}
$$

where the constant $C$ now depends on $\kappa$ and $d$.

And similarly for the sum over $\mathcal{P}_{0}$. 
In the next section the $L^{\infty}$-estimates (25) and (28), will be combined with the following Hölder regularity result shown in [9] Proposition 6.2 by Moser's iteration method for reversible random walks on infinite connected locally finite graphs with uniformly elliptic conductances:

Let $\left(a(e) ; e \in \mathcal{L}^{d}\right), d \geq 1$, be a (non-random) sequence of uniformly elliptic conductances. Then there are constants $\alpha>0$ and $C<\infty$, which depend only on the dimension and on the ellipticity constant, such that if for $N \geq 1, u: \bar{Q}_{2 N} \rightarrow \mathbb{R}$ verifies $\dot{H}_{2 N} u=0$ in $Q_{2 N}$, then for all $x, y \in Q_{N}$,

$$
|u(x)-u(y)|<C\left(\frac{|x-y|_{\infty}}{N}\right)^{\alpha} \max _{Q_{2 N}}|u|_{\infty}
$$

\subsection{Proof of Theorem 2}

Let $\left\{z_{i} ; 1 \leq i \leq d\right\}$ be the canonical basis of $\mathbb{R}^{d}$. For $1 \leq i \leq d$, let

$$
\dot{f}_{N}=N^{-d} z_{i}^{\prime} \dot{\mathcal{E}}_{N}\left(\dot{v}_{N}, \dot{v}_{N}\right) z_{i}
$$

where

$$
\dot{v}_{N}(x)=x+\dot{\chi}_{N}(x), \quad x \in \bar{Q}_{N} .
$$

Lemma 2. Let $\omega$ and $\sigma$ be two environments such that $a(e, \omega)=a(e, \sigma)$ for all edges e except maybe for $e=e_{k}$. Then for all $N \geq 1$,

$$
\left|\dot{f}_{N}(\omega)-\dot{f}_{N}(\sigma)\right| \leq \kappa N^{-d}\left(\dot{v}_{N}^{2}\left(e_{k}, \omega\right)+\dot{v}_{N}^{2}\left(e_{k}, \sigma\right)\right) .
$$

Proof of Lemma 2. By (24) and by 3a of Proposition 1, $\dot{v}_{N}$ is the solution of a variational problem. Then $\dot{f}_{N}(\omega)-\dot{f}_{N}(\sigma)$

$$
\begin{aligned}
& =N^{-d} \sum_{e} a(e, \omega)\left(z_{i} \cdot \dot{v}_{N}(e, \omega)\right)^{2}-N^{-d} \sum_{e} a(e, \sigma)\left(z_{i} \cdot \dot{v}_{N}(e, \sigma)\right)^{2} \\
& \leq N^{-d} \sum_{e}\left(\dot{a}_{N}(e, \omega)-\dot{a}_{N}(e, \sigma)\right)\left(z_{i} \cdot \dot{v}_{N}(e, \sigma)\right)^{2} \\
& \leq \kappa N^{-d} \dot{v}_{N}^{2}\left(e_{k}, \sigma\right) .
\end{aligned}
$$

Proof of Theorem 2. Let $\Delta_{k}=\mathbb{E}\left(\dot{f}_{N} \mid \mathcal{F}_{k}\right)-\mathbb{E}\left(\dot{f}_{N} \mid \mathcal{F}_{k-1}\right), k \geq 1$.

Let $M_{0}=0$ and $M_{n}=\sum_{1}^{n} \Delta_{k}, n \geq 1$. Then $\left(M_{n} ; n \geq 0\right)$ is a martingale and we will see that

$$
\dot{f}_{N}-\mathbb{E} \dot{f}_{N}=\sum_{1}^{\infty} \Delta_{k} \quad \text { a.s. and in } L^{2}
$$

We first check that $\left(M_{n} ; n \geq 0\right)$ verifies conditions (5) and (6).

By (25), (28) and the Hölder regularity (30), there are constants $\beta$ and $C<\infty$ which depend only on $\kappa$ and $d$ such that $\mathbb{P}$-a.s. and for all $N \geq 1$,

$$
\sup _{e} \dot{v}_{N}^{2}(e)<C N^{\beta}
$$


where $\beta=\min \{d-\alpha, 4-\alpha\}$. By Lemma 2 , we see that

$$
\begin{aligned}
\left|\Delta_{k}\right| & =\left|\mathbb{E}\left(\dot{f}_{N} \mid \mathcal{F}_{k}\right)-\mathbb{E}\left(\dot{f}_{N} \mid \mathcal{F}_{k-1}\right)\right| \\
& =\left|\mathbb{E}_{\sigma}\left(\dot{f}_{N}\left([\omega, \sigma]_{k}\right)-\dot{f}_{N}\left([\omega, \sigma]_{k-1}\right)\right)\right| \\
& \leq \mathbb{E}_{\sigma}\left|\dot{f}_{N}\left([\omega, \sigma]_{k}\right)-\dot{f}_{N}\left([\omega, \sigma]_{k-1}\right)\right| \\
& \leq \kappa N^{-d} \mathbb{E}_{\sigma}\left(\dot{v}_{N}^{2}\left(e_{k},[\omega, \sigma]_{k-1}\right)+\dot{v}_{N}^{2}\left(e_{k},[\omega, \sigma]_{k}\right)\right) \\
& \leq C N^{-d+\beta} .
\end{aligned}
$$

Hence, (6) holds with $B_{1}=C N^{-d+\beta}$. Similarly,

$$
\begin{aligned}
\Delta_{k}^{2} & \leq \mathbb{E}_{\sigma}\left(\left|\dot{f}_{N}\left([\omega, \sigma]_{k}\right)-\dot{f}_{N}\left([\omega, \sigma]_{k-1}\right)\right|^{2}\right) \\
& \leq 2 \kappa^{2} N^{-2 d} \mathbb{E}_{\sigma}\left(\dot{v}_{N}^{4}\left(e_{k},[\omega, \sigma]_{k-1}\right)+\dot{v}_{N}^{4}\left(e_{k},[\omega, \sigma]_{k}\right)\right) \\
& \leq C N^{-2 d+\beta} \mathbb{E}_{\sigma}\left(\dot{v}_{N}^{2}\left(e_{k},[\omega, \sigma]_{k-1}\right)+\dot{v}_{N}^{2}\left(e_{k},[\omega, \sigma]_{k}\right)\right) .
\end{aligned}
$$

Let

$$
U_{k}(\omega)=2 C N^{-2 d+\beta} \dot{v}_{N}^{2}\left(e_{k}, \omega\right) .
$$

We see that $\mathbb{E}\left(\Delta_{k}^{2} \mid \mathcal{F}_{k-1}\right) \leq \mathbb{E}\left(U_{k} \mid \mathcal{F}_{k-1}\right)$ and that (5) holds with $B_{0}=2 C N^{-d+\beta}$ since

$$
\begin{aligned}
\sum_{k} U_{k} & =2 C N^{-2 d+\beta} \sum_{k} \dot{v}_{N}^{2}\left(e_{k}, \omega\right) \\
& \leq C N^{-2 d+\beta} \operatorname{tr} \dot{\mathcal{E}}\left(\dot{v}_{N}, \dot{v}_{N}\right)_{N, \omega} \leq C N^{-2 d+\beta+d}=C N^{-d+\beta} .
\end{aligned}
$$

Then the martingale estimates I hold with $B=\max \left\{B_{0}, e B_{1}^{2}\right\}=B_{0}$. Hence, for all $t>0$,

$$
\mathbb{P}\left(\left|\dot{f}_{N}-\mathbb{E} \dot{f}_{N}\right| \geq t\right) \leq 4 \exp \left(-C t N^{(d-\beta) / 2}\right)
$$

and for all $N \geq 1, \operatorname{Var}\left(\dot{f}_{N}\right) \leq C N^{\beta-d}$.

\section{Spectral gap With Dirichlet Boundary CONDitions}

In this last example, we obtain tail estimates for the spectral gap of the random walk on an increasing sequence of cubes under Dirichlet boundary conditions.

Let $\mathcal{H}_{N, 0}=\left\{u: \bar{Q}_{N} \rightarrow \mathbb{R} ; u=0\right.$ on $\left.\partial Q_{N}\right\}$. If $u, v \in \mathcal{H}_{N, 0}$ then $\mathcal{E}_{N}(u, v)=(H u, v)_{N}$ where the Dirichlet form $\mathcal{E}_{N}$ is defined in (11).

Let $\psi_{N} \in \mathcal{H}_{N, 0}$ be the solution of the variational problem:

$$
\mathcal{E}_{N}\left(\psi_{N}, \psi_{N}\right)=\inf \left\{\mathcal{E}_{N}(u, u) ; u \in \mathcal{H}_{N, 0},\|u\|_{2, N}=1\right\} .
$$

Then $\psi_{N}$ is unique (up to a sign) and is an eigenfunction of $H$ acting on $\mathcal{H}_{N, 0}$. Let $\lambda_{N}>0$ be the corresponding eigenvalue. It was shown in [2], by homogenization methods as in Kesavan [17], that $N^{2} \lambda_{N}$ converges $\mathbb{P}$-a.s. and in $L^{1}(\mathbb{P})$ as $N \rightarrow \infty$ to the Dirichlet eigenvalue of a second-order elliptic operator with constant coefficients. 
The $L^{\infty}$ estimates of the eigenfunction is provided by the De Georgi-Nash-Moser theory (see [8] Sect. 2.1 and [7] Chap. 11):

Let a(e) be (non-random) uniformly elliptic conductances on the cubic lattice $\mathbb{Z}^{d}, d \geq 3$. Then there is a constant $C<\infty$ which depends only on the dimension and on the ellipticity constant such that if for $N \geq 1$, $\psi \in \mathcal{H}_{N, 0}$ is a normalized eigenfunction of $H$, that is, for some $\lambda>0, H \psi=\lambda \psi$ on $Q_{N}$, then for all $x \in Q_{N}$,

$$
|\psi(x)| \leq C \lambda^{d / 4} .
$$

Theorem 4. Let $\left(a(e) ; e \in \mathcal{L}^{d}\right)$ be a sequence of i.i.d. uniformly elliptic conductances on $\mathbb{Z}^{d}, d \geq 3$. Let

$$
A_{N}=N^{2} \lambda_{N}=N^{2} \mathcal{E}_{N}\left(\psi_{N}, \psi_{N}\right) .
$$

Then there is a constant $C<\infty$ which depends only on $d$ and $\kappa$ such that for all $t>0$ and $N \geq 1$,

$$
\mathbb{P}\left(\left|A_{N}-\mathbb{E} A_{N}\right| \geq t N^{(2-d) / 2}\right) \leq 4 \exp (-t / C)
$$

and

$$
\operatorname{Var}\left(A_{N}\right) \leq C N^{2-d} .
$$

Lemma 3. Let $\omega$ and $\sigma$ be two environments such that $a(e, \omega)=a(e, \sigma)$ for all edges e except maybe at $e=e_{k}$, an edge with endpoints $x_{k} \sim y_{k}$ of $\mathbb{Z}^{d}$, where

$$
a\left(e_{k}, \omega\right) \geq a\left(e_{k}, \sigma\right)
$$

Then for all $N \geq 1$,

$$
\left|A_{N}(\omega)-A_{N}(\sigma)\right| \leq C\left(N^{2} \psi_{N}^{2}\left(e_{k}, \sigma\right)+\psi_{N}^{2}\left(x_{k}, \omega\right)+\psi_{N}^{2}\left(y_{k}, \omega\right)\right) .
$$

Proof. Since the normalized eigenfunction $\psi_{N}(\omega)$ is a solution of a variational problem,

$$
\lambda_{N}(\omega)=\mathcal{E}_{N}\left(\psi_{N}(\omega), \psi_{N}(\omega)\right)_{\omega} \leq \gamma \mathcal{E}_{N}\left(\psi_{N}(\sigma), \psi_{N}(\sigma)\right)_{\omega}
$$

where $\gamma=\left\|\psi_{N}(\sigma)\right\|_{2, N, \omega}^{-2}$.

Furthermore, note that $\gamma \leq 1$ since $\left\|\psi_{N}(\sigma)\right\|_{2, N, \sigma}=1$ and $a\left(e_{k}, \omega\right) \geq a\left(e_{k}, \sigma\right)$.

Then

$$
\begin{aligned}
\lambda_{N}(\omega)-\lambda_{N}(\sigma)= & \mathcal{E}_{N}\left(\psi_{N}(\omega), \psi_{N}(\omega)\right)_{\omega}-\mathcal{E}_{N}\left(\psi_{N}(\sigma), \psi_{N}(\sigma)\right)_{\sigma} \\
\leq & \gamma \mathcal{E}_{N}\left(\psi_{N}(\sigma), \psi_{N}(\sigma)\right)_{\omega}-\mathcal{E}_{N}\left(\psi_{N}(\sigma), \psi_{N}(\sigma)\right)_{\sigma} \\
= & (\gamma-1) \mathcal{E}_{N}\left(\psi_{N}(\sigma), \psi_{N}(\sigma)\right)_{\omega} \\
& +\mathcal{E}_{N}\left(\psi_{N}(\sigma), \psi_{N}(\sigma)\right)_{\omega}-\mathcal{E}_{N}\left(\psi_{N}(\sigma), \psi_{N}(\sigma)\right)_{\sigma} \\
\leq & \mathcal{E}_{N}\left(\psi_{N}(\sigma), \psi_{N}(\sigma)\right)_{\omega}-\mathcal{E}_{N}\left(\psi_{N}(\sigma), \psi_{N}(\sigma)\right)_{\sigma} \\
\leq & \left(a\left(e_{k}, \omega\right)-a\left(e_{k}, \sigma\right) \psi_{N}^{2}\left(e_{k}, \sigma\right)\right. \\
\leq & \kappa \psi_{N}^{2}\left(e_{k}, \sigma\right) .
\end{aligned}
$$

Similarly, by the variational principle,

$$
\begin{aligned}
\lambda_{N}(\sigma)-\lambda_{N}(\omega) \leq & \bar{\gamma} \mathcal{E}_{N}\left(\psi_{N}(\omega), \psi_{N}(\omega)\right)_{\sigma}-\mathcal{E}_{N}\left(\psi_{N}(\omega), \psi_{N}(\omega)\right)_{\omega} \\
& \text { where } \bar{\gamma}=\left\|\psi_{N}(\omega)\right\|_{2, N, \sigma}^{-2} \\
= & (\bar{\gamma}-1) \mathcal{E}_{N}\left(\psi_{N}(\omega), \psi_{N}(\omega)\right)_{\sigma} \\
& +\mathcal{E}_{N}\left(\psi_{N}(\omega), \psi_{N}(\omega)\right)_{\sigma}-\mathcal{E}_{N}\left(\psi_{N}(\omega), \psi_{N}(\omega)\right)_{\omega} \\
\leq & (\bar{\gamma}-1) \mathcal{E}_{N}\left(\psi_{N}(\omega), \psi_{N}(\omega)\right)_{\sigma}
\end{aligned}
$$

since for all edges $a(e, \omega) \geq a(e, \sigma)$. 
Note that for all $u \in \mathcal{H}_{N, 0}$,

$$
0 \leq\|u\|_{2, N, \omega}^{2}-\|u\|_{2, N, \sigma}^{2} \leq a(e, \omega)\left(u^{2}\left(x_{k}\right)+u^{2}\left(y_{k}\right)\right)
$$

Then since $\psi_{N}(\omega)$ is a normalized eigenfunction and the conductances are uniformly elliptic,

$$
0 \leq \bar{\gamma}-1 \leq C\left(\psi_{N}^{2}\left(x_{k}, \omega\right)+\psi_{N}^{2}\left(y_{k}, \omega\right)\right)
$$

Then, $\lambda_{N}(\sigma)-\lambda_{N}(\omega) \leq C N^{-2}\left(\psi_{N}^{2}\left(x_{k}, \omega\right)+\psi_{N}^{2}\left(y_{k}, \omega\right)\right)$.

Proof of Theorem 4. As in the two preceding situations, we will verify conditions (5) and $(6)$ for $\Delta_{k}=\mathbb{E}\left(f_{N}\right.$ $\left.\mathcal{F}_{k}\right)-\mathbb{E}\left(f_{N} \mid \mathcal{F}_{k-1}\right)$.

$$
\begin{aligned}
\left|\Delta_{k}\right| \leq & \mathbb{E}_{\sigma}\left(\left|f_{N}\left([\omega, \sigma]_{k}\right)-f_{N}\left([\omega, \sigma]_{k-1}\right)\right|\right) \\
= & \mathbb{E}_{\sigma}\left(|\cdots| ; a\left(e_{k}, \omega\right) \geq a\left(e_{k}, \sigma\right)\right)+\mathbb{E}_{\sigma}\left(|\cdots| ; a\left(e_{k}, \omega\right)<a\left(e_{k}, \sigma\right)\right) \\
\leq & C \mathbb{E}_{\sigma}\left(N^{2} \psi_{N}^{2}\left(e_{k},[\omega, \sigma]_{k-1}\right)+\psi_{N}^{2}\left(x_{k},[\omega, \sigma]_{k}\right)+\psi_{N}^{2}\left(y_{k},[\omega, \sigma]_{k}\right)\right) \\
& +C \mathbb{E}_{\sigma}\left(N^{2} \psi_{N}^{2}\left(e_{k},[\omega, \sigma]_{k}\right)+\psi_{N}^{2}\left(x_{k},[\omega, \sigma]_{k-1}\right)\right. \\
& \left.+\psi_{N}^{2}\left(y_{k},[\omega, \sigma]_{k-1}\right)\right) \\
\leq & C N^{2-d}
\end{aligned}
$$

since by $(31),\left|\psi_{N}(x)\right| \leq C N^{-d / 2}$, for all $x \in Q_{N}$.

Pursuing the above calculations, and using (31) again, we find that

$$
\begin{aligned}
\Delta_{k}^{2} \leq & C N^{4-d} \mathbb{E}_{\sigma}\left(\psi_{N}^{2}\left(e_{k},[\omega, \sigma]_{k-1}\right)+\psi_{N}^{2}\left(e_{k},[\omega, \sigma]_{k}\right)\right) \\
& +C N^{-d} \mathbb{E}_{\sigma}\left(\psi_{N}^{2}\left(x_{k},[\omega, \sigma]_{k}\right)+\psi_{N}^{2}\left(y_{k},[\omega, \sigma]_{k}\right)\right. \\
& \left.+\psi_{N}^{2}\left(x_{k},[\omega, \sigma]_{k-1}\right)+\psi_{N}^{2}\left(y_{k},[\omega, \sigma]_{k-1}\right)\right)
\end{aligned}
$$

Let $U_{k}(\omega)=C N^{4-d} \psi_{N}^{2}\left(e_{k}, \omega\right)+C N^{-d}\left(\psi_{N}^{2}\left(x_{k}, \omega\right)+\psi_{N}^{2}\left(y_{k}, \omega\right)\right)$.

We see that $\mathbb{E}\left(\Delta_{k}^{2} \mid \mathcal{F}_{k-1}\right) \leq \mathbb{E}\left(U_{k} \mid \mathcal{F}_{k-1}\right)$ and

$$
\begin{aligned}
\sum_{k} U_{k} & <C N^{4-d} \sum_{k} \psi_{N}^{2}\left(e_{k}, \omega\right)+C N^{-d} \sum_{k}\left(\psi_{N}^{2}\left(x_{k}, \omega\right)+\psi_{N}^{2}\left(y_{k}, \omega\right)\right) \\
& =C N^{4-d} \mathcal{E}\left(\psi_{N}, \psi_{N}\right)_{\omega}+C N^{-d}\left\|\psi_{N}\right\|_{2, N}^{2} \\
& \leq C N^{4-d-2}+C N^{-d} \leq C N^{2-d}
\end{aligned}
$$

Hence (5) holds with $B_{0}=C N^{2-d}$ and (6) holds with $B_{1}=C N^{2-d}$. Since $B=\max \left\{B_{0}, e B_{1}^{2}\right\} \leq C N^{2-d}$, we obtain that for all $N \geq 1$ and $t>0$,

$$
\mathbb{P}\left(\left|f_{N}-\mathbb{E} f_{N}\right| \geq t\right) \leq 4 \exp \left(-\frac{t}{C} N^{(d-2) / 2}\right) \quad \text { and } \quad \operatorname{Var}\left(f_{N}\right) \leq C N^{2-d}
$$

Acknowledgements. The author is indebted to the referee for his many helpful comments. 


\section{REFERENCES}

[1] I. Benjamini and R. Rossignol, Submean variance bound for effective resistance on random electric networks. arXiv:math/0610393v4 [math.PR]

[2] D. Boivin and J. Depauw, Spectral homogenization of reversible random walks on $\mathbb{Z}^{d}$ in a random environment. Stochastic Process. Appl. 104 (2003) 29-56.

[3] D. Boivin and Y. Derriennic, The ergodic theorem for additive cocycles of $\mathbb{Z}^{d}$ or $\mathbb{R}^{d}$. Ergodic Theory Dynam. Syst. 11 (1991) 19-39.

[4] E. Bolthausen and A.S. Sznitman, Ten lectures on random media. DMV Seminar, Band 32, Birkhäuser (2002).

[5] A. Bourgeat and A. Piatnitski, Approximations of effective coefficients in stochastic homogenization. Ann. Inst. H. Poincaré Probab. Statist. 40 (2004) 153-165.

[6] P. Caputo and D. Ioffe, Finite volume approximation of the effective diffusion matrix: the case of independent bond disorder. Ann. Inst. H. Poincaré Probab. Statist. 39 (2003) 505-525.

[7] F.R.K. Chung, Spectral graph theory. CBMS Regional Conference Series in Mathematics, 92. American Mathematical Society (1997).

[8] E.B. Davies, Heat kernels and spectral theory. Cambridge Tracts in Mathematics, 92. Cambridge University Press (1989).

[9] T. Delmotte, Inéalité de Harnack elliptique sur les graphes. Colloq. Math. 72 (1997) 19-37.

[10] R. Durrett, Probability: Theory and Examples. Wadsworth \& Brooks/Cole Statistics/Probability Series (1991).

[11] L.R.G. Fontes and P. Mathieu, On symmetric random walks with random conductances on $\mathbb{Z}^{d}$. Probab. Theory Related Fields 134 (2006) 565-602.

[12] T. Funaki and H. Spohn, Motion by mean curvature from the Ginzburg-Landau $\nabla \phi$ interface model. Commun. Math. Phys. 185 (1997) 1-36.

[13] G. Grimmett, Percolation. 2nd ed. Springer (1999).

[14] E. Hebey, Nonlinear analysis on manifolds: Sobolev spaces and inequalities. Courant Lecture Notes Mathematics 5. American Mathematical Society (2000).

[15] B.D. Hughes, Random walks and random environments. Vol. 2. Random environments. Oxford University Press (1996).

[16] V.V. Jikov, S.M. Kozlov and O.A. Olejnik, Homogenization of differential operators and integral functionals. Springer-Verlag (1994).

[17] S. Kesavan, Homogenization of elliptic eigenvalue problems I. Appl. Math. Optimization 5 (1979) 153-167.

[18] H. Kesten, On the speed of convergence in first-passage percolation. Ann. Appl. Probab. 3 (1993) 296-338.

[19] C. Kipnis and S.R.S. Varadhan, Central limit theorem for additive functionals of reversible Markov processes and applications to simple exclusions. Comm. Math. Phys. 10 (1986) 1-19.

[20] S.M. Kozlov, The method of averaging and walks in inhomogeneous environments. Russ. Math. Surv. 40 (1985) $73-145$.

[21] R. Kuennemann, The diffusion limit for reversible jump processes on $Z^{m}$ with ergodic random bond conductivities. Commun. Math. Phys. 90 (1983) 27-68.

[22] H. Owhadi, Approximation of the effective conductivity of ergodic media by periodization. Probab. Theory Related Fields 125 (2003) 225-258.

[23] E. Pardoux and A.Yu. Veretennikov, On the Poisson equation and diffusion approximation. I. Ann. Probab. 29 (2001) 10611085 .

[24] Y. Peres, Probability on trees: An introductory climb. Lectures on probability theory and statistics. École d'été de Probabilités de Saint-Flour XXVII-1997, Springer. Lect. Notes Math. 1717 (1999) 193-280 .

[25] L. Saloff-Coste, Lectures on finite Markov chains. Lectures on probability theory and statistics. École d'été de probabilités de Saint-Flour XXVI-1996, Springer. Lect. Notes Math. 1665 (1997) 301-413.

[26] V. Sidoravicius and A.-S. Sznitman, Quenched invariance principles for walks on clusters of percolation or among random conductances. Probab. Theory Related Fields 129 (2004) 219-244.

[27] F. Spitzer, Principles of random walk. The University Series in Higher Mathematics. D. Van Nostrand Company (1964).

[28] J. Wehr, A lower bound on the variance of conductance in random resistor networks. J. Statist. Phys. 86 (1997) 1359-1365.

[29] V.V. Yurinsky, Averaging of symmetric diffusion in random medium. Sib. Math. J. 2 (1986) 603-613. 